\title{
The distribution of subspecialties in ophthalmology group practices in an era of change
}

\section{Introduction}

The last 25years have seen enormous changes in the way healthcare is delivered. The pace of change has accelerated rapidly in the most recent decade. Hospitals, insurers, pharmaceutical companies, academic centers and physicians have all evolved to adapt to this new environment. ${ }^{1-14}$ Expansion, mergers, consolidation of services, and the formation of satellite offices are some of these adaptations. ${ }^{15-17}$ Optimizing resource allocation is more vital than ever.

Ophthalmology has been particularly impacted by demographic shifts that are unfolding in the United States. The elderly population is expanding. Every day 10,000 of the 78 million baby boomers are turning 65 , and individuals aged 65 and older require about ten times as much ophthalmic care..$^{5}$ Age related diseases including cataracts, macular degeneration, glaucoma, and diabetic retinopathy are increasing at approximately 5\% per year. ${ }^{5}$ Not only has this cohort expanded, but this trend is expected to continue for the next 30 years. ${ }^{5}$ The cost of medical equipment is particularly high in the field of Ophthalmology and reimbursements for services are expected to decrease, putting tremendous pressure on practices to handle these challenges. ${ }^{4,5,16}$ It is difficult to determine how to staff a multi-subspecialty practice in this environment. The decision to hire a subspecialist involves weighing many factors including patient demand, reimbursement, the availability of physician applicants, competitive salaries, and the synergistic effects that the new physician may have on the group practice.

The primary aim of this study is to describe how academic ophthalmology group practices in the United States allocate worktime to their various subspecialties. The estimated work-time allocation of subspecialists may be useful as a rough baseline guide for those forming a new practice or expanding a current practice. This study was conducted as a follow-up to a previous resource allocation study to reexamine the distribution of subspecialists in academic ophthalmology group practices. ${ }^{18}$ It seemed reasonable to revisit this subject because of the widespread changes that are taking place in healthcare in general, and in ophthalmology in particular.

Ophthalmology is well suited for this study because it contains numerous sub-specialties. We chose academic centers because they are independent groups that provide a wide variety of subspecialty care. The Web sites of the same 40 medical schools in our previous study were examined for information on the following subspecialties: comprehensive care, retina, glaucoma, cornea, oculoplastics, pediatric ophthalmology, neuro-ophthalmology, and uveitis. To our knowledge, there are no other workforce allocation studies in the literature that focus on the level of multi-subspecialty group practices in ophthalmology. In addition, the methodology used in this study is generalizable and may be applied to any field of medicine that has multiple subspecialties.

Finally, the study has relevance to federal medicine because of the close affiliations nationwide of academic centers with Department of Veterans Affairs (VA) Medical Centers.
Volume 8 Issue 5 - 2018

\author{
Margaret M Palmisano,' Philip C Palmisano \\ 'Biostatistician, MS Biostatistics, PhD Biology, USA \\ ${ }^{2}$ Assistant Professor of Ophthalmology, Yale University School of \\ Medicine, USA
}

Correspondence: Philip C Palmisano, Chief of Ophthalmology, VA Connecticut Healthcare System, Assistant Professor of Ophthalmology, Yale University School of Medicine, USA, Tel (203) 4150709, Email philip.palmisano@yale.edu

Received: August 18, 2018| Published: September 06, 2018

\section{Methods}

Subspecialty data on each full-time physician from the web sites of 40 Medical Schools was examined from 7 September 2016 to 1 October 2016. We considered 8 subspecialties of ophthalmology in this study: comprehensive ophthalmology, retina/vitreous, pediatric ophthalmology, cornea/external disease, glaucoma, oculoplastics, neuro-ophthalmology, and uveitis. Comprehensive ophthalmology is the broadest clinical area and includes everything from complete eye exams to anterior segment surgery (cataract surgery) and various laser procedures.

All of the methods used in this study are identical to those used in our prior study. ${ }^{18}$ Collection of data, sampling methods, statistical methods, Full Time Equivalent Employee (FTEE) coding methods, as well as study limitations are fully explained in detail therein. It should be noted that the 40 practices analyzed in this study are the same 40 practices analyzed in our last study. ${ }^{18}$

The basic measure used in this study is the amount of work-time that is allocated to an employee, which is quantified in units called FTEE. In this study, 1 FTEE represents a working schedule of 40hours per week. Any fraction of FTEE can be translated into hours per week. For example, 0.5 FTEE is equivalent to 20 hours of work per week. Since actual FTEE is not presented on the websites of the medical schools we estimated FTEE based on information provided on the websites. Available information on fellowship training, selfreported clinical care and interests, as well as departmental listings of physicians under the various subspecialty divisions were used to estimate FTEE values.

All statistical analyses and graphics were performed using $\mathrm{R}$ software (version 3.0.2). ${ }^{19}$ Cluster analysis was performed using the K-means clustering algorithm of Hartigan and Wong. ${ }^{20}$ Practices with similar proportions of FTEE for each of the 8 subspecialties were assigned to the same cluster, whereas practices with different proportions of FTEE were assigned to different clusters. A large homogeneous cluster would suggest the existence of a "typical" or average practice. The mean proportion of FTEE for each subspecialty 
in this cluster could then be used to describe the work-time allocation of a typical ophthalmology practice.

Data on the number of satellite clinics for each of the 40 practices was collected from 7 May 2017 to 25 August 2017 from the websites of the schools. Satellite clinics are generally smaller offices located in sites other than the large main academic site.

\section{Results}

We collected subspecialty data on 946 full-time physicians from 40 Medical Schools in this study. Ophthalmology departments ranged from 4 to 89 physicians. The average size of a practice was 24 and the median was 16 .

\section{Missing Subspecialties}

A subspecialty could be missing for 2 reasons. 1) no physicians were listed under that particular subspecialty or 2) the physicians that were listed under the subspecialty were classified under part-time status. In practices that contained fewer than 8 subspecialties, uveitis and neuro-ophthalmology were missing most frequently. Table 1

Table I Number of missing subspecialties $(\mathrm{N}=40)^{\mathrm{a}}$

\begin{tabular}{ll}
\hline Practices with missing & \\
\hline Subspecialty & Sub speciialties \\
\hline & Number $(\%)$ \\
Comprehensive care & $0(0.00)$ \\
Retina & $2(0.05)$ \\
Glaucoma & $3(0.08)$ \\
Pediatric Ophthalmology & $4(0.10)$ \\
Oculoplastics & $4(0.10)$ \\
Neuro-Ophthalmology & $5(0.12)$ \\
Uveitis & $7(0.18)$ \\
\hline
\end{tabular}

${ }^{\text {a }}$ Please refer to the results section for the definition of missing subspecialties used in this study

Of the 40 practices, 14(35\%) did not include uveitis and 7(18\%) did not include neuro-ophthalmology. Conversely, comprehensive was included in all of the practices, cornea was absent in only 2 practices and retina was absent in only 3 practices.

\section{Mean proportion of FTEE by subspecialty and practice characteristics}

Table $2 \mathrm{~A}$ presents the mean proportion FTEE by subspecialty for all 40 practices (pooled) and for practices classified by region. ${ }^{21}$ Corresponding characteristics are presented in Table 2B.

\section{Mean proportion of FTEE by subspecialty (Pooled estimates)}

Based on proportion FTEE data of the 40 practices, comprehensive has the highest mean proportion of FTEE (0.352) followed by retina $(0.180)$. The next largest mean proportions of FTEE are pediatrics, glaucoma, cornea, and oculoplastics which have similar average proportions of FTEE, with values of around 0.100. Neuroophthalmology and uveitis have the smallest average proportion of FTEE with values of 0.026 and 0.019 , respectively (Table 2A).

\section{Description of 40 practices by geographical region $(\mathrm{N}=40)$}

The geographical distribution of the practices is as follows: 7 practices $(17.5 \%)$ in the Northeast; $7(17.5 \%)$ in the West; $10(25 \%)$ in the Midwest and 16(40\%) in the South.

The average number of physicians per practice across the 4 regions ranged from 20 to 27 physicians. The median number of physicians ranged from 12 to 28 physicians per practice.

\section{Mean proportion of FTEE by subspecialty (Regional estimates)}

The mean proportion of FTEE for each subspecialty by region are reported in Table 2A. The 4 regions are all similar in their ranking of comprehensive care as having the highest proportion of FTEE. Retina is ranked as the second highest in all regions except for the Midwest where it is ranked as having the third highest proportion of FTEE. Uveitis and neuro-ophthalmology have the lowest mean proportion of FTEE values. Uveitis is ranked as having the lowest values in 3 of the 4 regions, and Neuro-ophthalmology is ranked as having the second lowest values in 3 of the 4 regions. All other subspecialties have different intermediate rankings depending on the geographic region.

Table 2A Mean Proportion of FTEE by Subspecialty $(\mathrm{N}=40)$

\begin{tabular}{lllllllll}
\hline Practices & \multicolumn{2}{l}{ Subspecialty } & & & & & \\
\hline & Comp & Retina & Pediatric & Glaucoma & Cornea & Oculo & Neuro & Uveitis \\
\hline Pooled $^{\mathrm{a}}$ & 0.352 & 0.18 & 0.126 & 0.108 & 0.104 & 0.086 & 0.026 & 0.019 \\
Region $^{\mathrm{b}}$ & & & & & & & & \\
NE & 0.381 & 0.191 & 0.071 & 0.096 & 0.114 & 0.103 & 0.022 & 0.023 \\
MW & 0.294 & 0.157 & 0.165 & 0.146 & 0.094 & 0.093 & 0.035 & 0.017 \\
S & 0.383 & 0.183 & 0.11 & 0.095 & 0.115 & 0.08 & 0.018 & 0.016 \\
W & 0.334 & 0.193 & 0.164 & 0.096 & 0.081 & 0.073 & 0.034 & 0.026
\end{tabular}

Comp=comprehensive care; FTEE=full-time equivalent employee; $M W=$ midwest; Neuro=neuro-ophthalmology; $N E=$ northeast; Oculo=oculoplastics; $\mathrm{S}=$ south; West $=$ west.

${ }^{\text {a }}$ Pooled refers to all 40 practices

'The 4 regions are based on the U.S. Census Bureau's partitioning of the United States ${ }^{21}$

The Northeast consists of the New England states (CT, ME, MA, NH, RI,VT) and the Middle Atlantic states (NJ, NY, PA). The Midwest consists of the East North Central states (IN, IL, MI, OH,WI) and the West North Central states (IA, KS, MN, MO, NE, ND, SD). The South consists of the South Atlantic states (DE, DC, FL, GA, MD, NC, SC,VA, WV). The East South Central states (AL, KY, MS, TN), and the West South Central states (AR, LA, OK, TX). The West consists of the Mountain states (AZ, CO, ID, NM, MT, NV, UT,WY) and the Pacific states (AK, CA, HI, OR,WA) 
Table 2B Characteristics of 40 practices pooled and sub classified by region

\begin{tabular}{lllllll}
\hline Practices $^{\mathbf{a}}$ & \multicolumn{2}{l}{ Number of physicians } & & \\
\hline & Total & Average & Median & Minimum & Maximum & Total $^{\text {b }}$ \\
\hline Pooled & 40 & 23.65 & 16 & 4 & 89 & 946 \\
Regional & & & & & & \\
NE & 7 & 23.14 & 12 & 6 & 69 & 162 \\
MW & 10 & 19.9 & 19.5 & 9 & 30 & 199 \\
S & 16 & 24.88 & 15 & 4 & 89 & 398 \\
W & 7 & 26.71 & 28 & 9 & 53 & 187
\end{tabular}

$M W=$ midwest; $N E=$ northeast; $S=S o u t h ; W=$ west

aPlease refer to the legend of Table $2 a$ (footnotes $a$ and $b$ )

${ }^{\text {b}}$ Total number of physicians refers to the total number of full-time physicians studied

\section{Mean proportion FTEE using cluster analysis}

$\mathrm{K}$-means was done on the square roots of the proportion data to account for the differences in magnitude of variables with small counts such as uveitis and neuro-ophthalmology (Table 1). The analysis consisted of 1000 random sets of 3 distinct practices. The resulting clusters of practices are reported in Table $3 \mathrm{~A}$. Corresponding characteristics of the practices from each cluster are given in Table $3 \mathrm{~B}$.

Table 3A Mean proportion FTEE per subspecialty by cluster $^{\mathrm{a}}$

\begin{tabular}{lllllllll}
\hline Cluster $^{\mathrm{b}}$ & \multicolumn{2}{l}{ Subspecialty } \\
& Comp & Retina & Pediatric & Glaucoma & Cornea & Oculo & Neuro & Uveitis \\
\hline Large $(n=29)$ & 0.31 & 0.198 & 0.12 & 0.112 & 0.109 & 0.098 & 0.03 & 0.023 \\
Small $(n=8)$ & 0.399 & 0.152 & 0.197 & 0.135 & 0.061 & 0.042 & 0.011 & 0.003 \\
Smallest $(n=3)$ & 0.629 & 0.077 & 0 & 0 & 0.164 & 0.081 & 0.022 & 0.027
\end{tabular}

Comp=comprehensive care; FTEE=full-time equivalent employee; neuro=neuro-ophthalmology; oculo=oculoplastics; pediatric=pediatric ophthalmology

${ }^{a}$ The distribution of the mean proportion FTEE values in the large cluster $(n=26)$ represents the composition of a typical ophthalmology practice in our study ${ }^{b} n=$ number of practices in a cluster

Table 3B Characteristics of the clusters

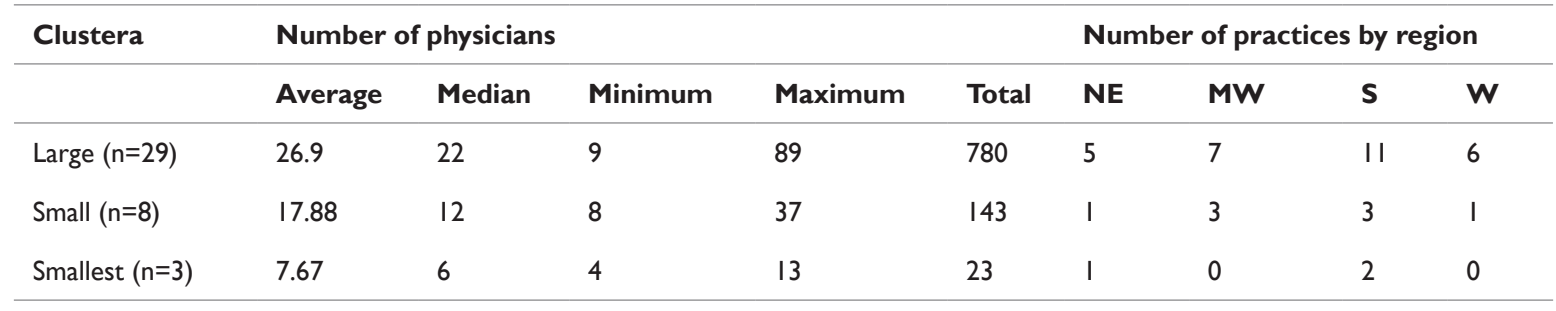

$M W=$ midwest $\mathrm{NE}=$ northeast $\mathrm{S}=$ south; $\mathrm{W}=$ west

${ }^{\mathrm{a}} \mathrm{n}=$ number of practices in a cluster

One dominant cluster, consisting of 29 practices, and 2 smaller clusters, composed of 8 practices and 3 practices, are present. The average number of physicians in the largest cluster was 27 physicians, and the average number of physicians in the clusters composed of 8 and 3 practices were 18 and 8 , respectively.

The geographic distributions of the largest cluster and the small cluster $(\mathrm{N}=8)$ resemble the regional sampling distribution of the pooled data. In contrast, the smallest cluster $(\mathrm{N}=3)$ is limited to 2 practices in the south and one practice in the northeast.

The members of the largest homogeneous cluster composed of 29 practices are considered to be representative of a typical ophthalmology practice in their allocation of FTEE to the 8 subspecialties. Values of the mean proportion of FTEE for the 8 subspecialties in this cluster are ranked as follows: comprehensive, retina, pediatric, glaucoma, cornea, oculoplastics, neuro-ophthalmology, and uveitis. Comprehensive had the highest proportion of FTEE (0.310) followed by Retina (0.198). The lowest proportions of subspecialists were neuro-ophthalmology (0.030) and uveitis (0.023). The remaining 4 subspecialties have about the same average proportion of FTEE with values of about 0.100 (Table 3A). 
Of the practices in the largest cluster (21/29), 72\% are composed of all 8 subspecialties. $27 \%(5 / 29)$ have only one subspecialty missing. Uveitis was absent in four of those 5 practices with one missing subspecialty. Glaucoma is absent in one of the 5 practices. Three practices in the largest cluster (3/29), 10\% had 2 subspecialties missing. Uveitis and neuro-ophthalmology are absent in two of these practices, and uveitis and pediatrics are absent in one.

The smaller cluster $(\mathrm{N}=8)$ is composed of 8 practices and the mean proportion of FTEE from highest to lowest values were as follows: comprehensive, pediatrics, retina, glaucoma, cornea, oculoplastics, neuro-ophthalmolgoy and uveitis. Comprehensive has the highest value of 0.399 , followed by pediatrics with a value of 0.197 . Retina and glaucoma had similar values of 0.152 and 0.135 . Neuroophthalmology and uveitis have the lowest mean proportion FTEE with values of 0.011 and 0.003 , respectively. Only one of the practices in this cluster had all of the 8 subspecialties represented. The number of missing subspecialties per practice in this cluster range from 1 to 4. Uveitis, oculoplastics, neuro-ophthalmology, cornea and retina are missing in $6,4,4,2$, and 1 practice, respectively.

The smallest cluster is composed of 3 practices and does not include any pediatric and glaucoma specialists. The mean proportion of FTEE listed from highest to lowest is as follows: comprehensive, cornea, oculoplastics, retina, uveitis, and neuro-ophthalmlogy. Comprehensive had the highest proportion of FTEE of 0.629 and cornea has the second highest proportion of 0.164 . The FTEE values in the other subspecialties in this cluster are less than 0.100 . None of the practices in this cluster contain all 8 subspecialties. The number of missing subspecialties in this cluster range from 2 to 5 . In addition to the absence of glaucoma and pediatrics, retina and neuro-ophthalmology are absent in the second practice and retina, oculoplastics and uveitis are absent in the third practice.

Cluster analysis elucidates 3 different types of practices.

The typical practice (largest Cluster) has the full spectrum of 8 subspecialties. The second smallest cluster is distinct from the typical practice primarily due to its high proportion of pediatrics. The smallest cluster is distinguished from the others based on its predominant proportion of Comprehensive care providers (0.629) and its complete absence of glaucoma and pediatric specialists. The latter finding suggests that small practices have difficulty in filling all of the 8 subspecialties.

\section{Distribution of the typical practice at 2 time points (2009 and 2016)}

Figure 1 presents the allocation of FTEE for the typical practice based on prior data collected in $2009^{18}$ and on current data collected in 2016 for this study. The mean distribution of FTEE is remarkably similar at these two points in time.

\section{Seven year turnover of the number of physicians (2009-2016)}

An estimate of the turnover of physicians was conducted using data collected in 2009 in our first study as the baseline reference. ${ }^{18}$

\section{Pooled data}

In terms of the total set of 40 practices in the pooled data, the net increase in full-time physicians was 30\% (220/726) from 2009 to 2016. This involved the gain of 475 new full-time physicians and the loss of 255 full-time physicians.

\section{Largest cluster (the typical practice in 20 I 6)}

The largest cluster was composed of 29 practices which were used to characterize the typical practice in 2016. The turnover in the typical practice is similar to that of the pooled data since a predominant number of practices and physicians in this study are members of the typical practice. More specifically, $76 \%$ of the total number of practices (29/40) and $82 \%$ of the total number of physicians (780/946) are present in the largest cluster. The 7year (2009-2016) net increase of physicians in the typical practice ( $\mathrm{N}=29$ practices) was $30 \%$ (181/599). The net increase of full-time physicians involved the addition of 382 and the loss of 201 individuals

\section{Composition and turnover of practices in the largest cluster}

$76 \%(22 / 29)$ of the practices in the largest cluster in the 2009 data remained in the largest cluster in 2016. Moreover, 7 of the 29 practices converged to the distribution of subspecialties in the typical practice. (Note: 15\% (4/26) of the practices in largest cluster in the 2009 study diverged from the typical practice allocation, and were not members of the 2016 largest cluster).

\section{Satellite data}

\section{Pooled data (data on all 40 practices)}

$72 \%(29 / 40)$ percent of the 40 practices have one or more satellite offices. The average number of satellites in the current study is 3 with a median of 2 . The range is from zero to 18 .

\section{The largest Cluster (typical practice)}

$72 \%(21 / 29)$ percent of the 29 practices in the typical practice have one or more satellite offices. The mean, median, and range for the typical practice in our current study are the same as those for the pooled data above, with all values rounded.

\section{Multiple fellowships}

We examined the available fellowship data on the 946 physicians in our study and discovered that multiple fellowships are fairly frequent. Among all sub-specialties, 5\% of physicians (51/946) have completed two fellowships. For specialists in the fields of uveitis and neuro-ophthalmology, dual fellowships are far more common. $49 \%$ of uveitis specialists and $30 \%$ of neuro-ophthalmology specialists had completed two fellowships. 90\% (46/51) of all of the dual fellowships in this study included uveitis or neuro-ophthalmology.

\section{Discussion}

Allocation of FTEE among the eight ophthalmology subspecialties is not an easy task. Clinical demands, reimbursements, budgetary constraints, availability of subspecialists, the amount of time and labor for procedures, the cost of new state of the art equipment, the emergence of new treatments, demographic shifts in the population, and the specific mission of the department are important factors to take into account when determining how to staff a practice. Ideally, tertiary referral practices can provide the most complete integrated eye care when all of the subspecialties are included.

The typical academic ophthalmology practice in our study contains the full spectrum of 8 subspecialties and has an average practice size of 27 physicians. Four levels of allocation are apparent in the typical practice. Comprehensive care has the highest allocation and falls into the first tier. Retina occupies the second tier. Pediatrics, glaucoma, 
cornea, and oculoplastics comprise the third tier and the lowest tier of all contains neuro-ophthalmology and uveitis.

The high level of FTTE for comprehensive care may due to its broad scope of treatment across a wide spectrum of eye diseases as well as its pivotal role as a point of triage to other subspecialties. Cataract surgery is in great demand because the disease is the most prevalent in the elderly cohort. ${ }^{22,23}$ The availability of comprehensive care providers is the highest among all of the areas of care in ophthalmology because all subspecialists must first complete their training as general ophthalmologists before entering fellowship training. In fact, many subspecialists divide their work-time between comprehensive care and their subspecialty.

The substantial allocation to retina may be attributed to the high incidence of treatable diseases as well as to the high reimbursements ${ }^{24}$ in this field.

Macular degeneration, and diabetic retinopathy are the second and third most common age-related ocular diseases. ${ }^{22,23}$

Even though each subspecialty in the third tier has a unique set of characteristics, they were found to have approximately the same work-time allocation. Glaucoma, which primarily provides care for the elderly, might be expected to have a relatively higher clinical demand because glaucoma is the fourth most common diseases in an aging population. ${ }^{22,23}$ Cornea serves a somewhat younger demographic than glaucoma. Pediatric ophthalmology provides care for the youngest cohort. It involves time intensive patient care with relatively low reimbursements. ${ }^{24}$ In contrast, the field of oculoplastics tends to have higher reimbursements ${ }^{24}$ particularly for cosmetic and elective procedures and treats patients across all age groups. It is clear from our findings that no one single factor was used to determine the allocation of FTEE in this tier. Clinical demand, the age-population structure, competitive salaries, and the incidence of treatable diseases are some of the many factors that influence staffing decisions.

The extremely low allocation for uveitis and neuro-ophthalmolgy may be explained by the labor intensive care of complex cases, the modest reimbursements ${ }^{24}$ and the relatively low incidence of disease in these subspecialties. ${ }^{25-27}$

A striking similarity is apparent when comparing the distribution of subspecialties of the typical practice in this study with the distribution in our prior study (Figure 1). The same four tiers of values for the mean proportion of FTEE exist in both studies. It is important to emphasize that the proportions of FTEE for the 8 subspecialties were similar in magnitude at both time points despite a substantial turnover of full time physicians. The net increase of fulltime physicians in the typical practice ( $\mathrm{N}=29$ practices) was $30 \%$ (181/599) involving the addition of 382 and the loss of 201 physicians. These results suggest that the academic group practices have found a way to meet clinical needs, while also responding to economic realities, incentives and limitations. $49 \%$ of physicians in the largest cluster $(\mathrm{N}=29)$ were new $(382 / 780)$ suggesting that when new physicians were hired, the proportionate allocation remained the same.

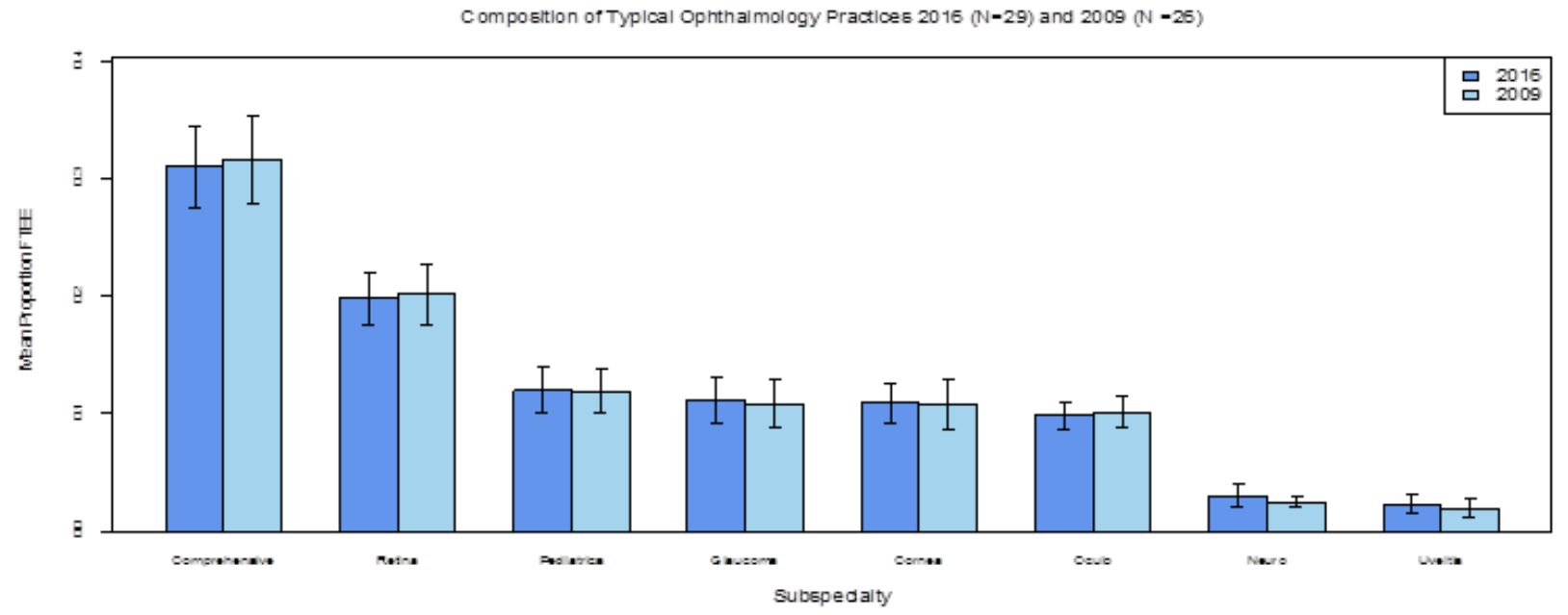

Figure I A comparison of the composition of a typical ophthalmology practice from data collected in 2016 to the composition of a typical ophthalmology practice from data collected in 2009. FTEE = full-time equivalent employee; neuro=neuro-ophthalmology; oculo=oculoplastics. Error bars represent $95 \%$ confidence intervals for the mean FTEE proportion for each subspecialty.

In addition, with an overall $30 \%$ net increase of physicians, a greater number of practices (29 practices in $2016 \mathrm{vs}^{26}$ practices in 2009) converged to the distribution of subspecialties in the typical practice. This may indicate the viability and putative benefits of the composition of the typical practice.

Two possible factors that might contribute to the preservation of this distribution of subspecialties may be the flexible nature of fellowship training and the utilization of satellite clinics.

The fellowship system is the primary source of new ophthalmology subspecialists and responds well to changes in healthcare demands. ${ }^{28}$
It has been observed that while it is a long, detailed process to expand ophthalmology residency positions to increase the overall supply of ophthalmologists, ${ }^{29}$ it is easier to expand fellowship positions to create new sub-specialists in fields such as retina, glaucoma or cornea. While residencies are threeyears long, with extensive training costs and regulatory limitations, a fellowship position usually only takes one or rarely two years, and can even be created by a private practice.

Dual fellowships may contribute to the retention of uveitis and neuro-ophthalmology subspecialties in the typical practice. We examined the available fellowship data on the 946 physicians in our study. $5 \%$ of the physicians in our study had completed two 
fellowships. $90 \%$ of the dual fellowships included uveitis or neuroophthalmology, subspecialties that are time and labor intensive with modest reimbursements. By combining them with the relatively better compensated sub-specialties such as retina or oculoplastics, a single physician can diversify his practice to serve patients who would otherwise have greater difficulty finding care. ${ }^{30}$ There have been concerns over the last several years that these sub-specialties would become less common. ${ }^{31}$ Perhaps partly due to the phenomenon of dual fellowships, our study suggests that these subspecialties have endured.

Satellite offices may play an important role in allowing practices to maintain their full sub-specialty profile. They expand a department's geographical reach and increase patient volume, ${ }^{15}$ and also improve access to care. Because not all sub-specialties tend to be represented, ${ }^{15,16}$ satellites may provides a large enough patient base to support the sub-specialists at the main academic center, particularly those who treat less common diseases such as neuro-ophthalmologists and uveitis specialists. $72 \%$ of the practices in this study had satellite locations. It has been reported that in one very large practice, $80 \%$ of the total patient volume, and $50 \%$ of the total patient revenue were attributed to the satellites. ${ }^{32}$

One notable difference between the typical practice of 2006 and the typical practice of 2016 is the increase in the average number of physicians per practice from 23 to 27 . This change is consistent with a response to a growing demand for eye care. Some recent estimates suggest that "The demand for eye care is soaring at about five times the U.S. population growth..."2 due to the increase in the older patient cohort. Larger practices may not only benefit patients by providing a wider variety of services, and improved continuity of care, but may also potentially benefit physicians through cost efficiency from shared overhead and cross-referrals. ${ }^{33-35}$ Despite the increase in the average number of physicians per practice, the allocation of FTEE in the typical practice from the 2009 study was retained suggesting the feasibility of this distribution as a rough guide for staffing.

In summary, our study found a robust allocation of FTEE across the subspecialties in the typical practice and an increase in the average number of physicians in the typical practice. The increase in practice size may be primarily attributed to and enabled by demographic changes in the population. Possible reasons for the stability of FTEE allocation may be explained in part by the use of satellite offices and flexible and timely opportunities for fellowship training.

Since only two time points were used in this study, one cannot confidently draw conclusions about a trend toward this distribution, because we do not know the distributions for any of the intervening years. However, the remarkable similarity in the distribution of FTEE separated by 7years and accompanied by a high turnover of physicians strongly suggests that the distribution of a typical practice has remained stable.

\section{Conclusion}

The results of the K-means cluster analysis of 40 academic group practices suggest that the typical practice may be characterized as being composed of the following proportions of subspecialties listed in order of the largest to the smallest Comprehensive (0.310), retina (0.198), pediatrics $(0.120)$ glaucoma $(0.112)$, cornea $(0.109)$, oculoplastics (0.098), neuro-ophthalmology (0.03) and uveitis (0.023). This distribution can be used by any practice as a rough, baseline guide for FTEE allocation. Perhaps the best overall conclusion is that despite major changes in the healthcare system, the composition of the typical academic ophthalmology group practice has remained remarkably stable and continues to find a way to fulfill its important role in preserving the availability of vital tertiary services, and training the next generation of specialists and subspecialists. The availability of subspecialists in academic centers is particularly important for VA Medical Centers, which have had a long and successful history of collaboration with academic centers for the shared missions of patient care and physician education.

\section{Acknowledgements}

None.

\section{Conflict of interest}

The author declares no conflict of interest.

\section{References}

1. Duker JS, Graber CJ, Lindstrom RL, et al. Private equity offers new option for ophthalmology practice consolidation. Ocul Surg News. 2017;35(11):10-12.ÿ

2. Pinto JB. Heads or tails: Will headwinds or tailwinds prevail for private equity firms buying ophthalmology practices? Ocul Surg News. 2017:35(17):16.

3. Chari R, O'hanlon $\mathrm{C}$, Chen $\mathrm{P}$, et al. Governing academic medical center systems: Evaluating and choosing among alternative governance approaches. Acad Med. 2018;93(2):192-198.

4. Winslow R. Mayo's tricky task: Revamp what works. Wall St J. 2017;3-4:A1,A10.

5. Kent C. Preparing your practice for the senior tsunami. Rev Ophthalmol. 2016;23(5):26-34.

6. Kent C. Coming together: Is a bigger practice better? Rev Ophthalmol. 2016;23(10):32-41.

7. Academic medical centers: Trends in mergers, acquisitions and affiliations. Becker's Hospital Review. 2016.

8. Block S, Sonnino RE, Bellini L. Defining "faculty" in academic medicine: responding to the challenges of a changing environment. Acad Med. 2015;90(3):279-282.

9. Their SO, Kelley WN, Pardes H, et al. Success factors in merging teaching hospitals. Acad Med. 2014;89(2):219-223.

10. Kent C. Can a comprehensive practice still survive? Rev of Ophthalmol. 2014;21(10):30-39.

11. Kahn MJ, Maurer R, Wartman SA, et al. A case for change: disruption in academic medicine. Acad Med. 2014;89(9):1216-1219.

12. Coulson K. Using data to add a new practice site. How demographics can drive a fact-based approach. Ophthalmol Manage. 2014:18.

13. Mets MB, Rich WL, Lee P, et al. The ophthalmic practice of the future. Ach Ophthalmol. 2012;130(9):1195-1198.

14. Billi JE, Wise CG, Middleton SC, et al. Financial impact of the medicare fee schedule on a large multispecialty faculty practice in an academic medical center. Acad Med. 1993;68(5):315-322.

15. Kuo IC. Satellite clinics in academic ophthalmology programs an exploratory study of successes and challenges. BMC Ophthalmol. 2013;13:79.

16. Evans M. Hospitals invest in outside clinics. Wall St J. 2017. 
17. Kuo IC, Wright SM. Impact of satellite practices on academic ophthalmology departments. J Acad Ophthalmol. 2017;9(1):e7-e12.

18. Palmisano PC, Palmisano MM. The distribution of subspecialties in ophthalmology group practices. Fed Pract. 2011; 28(11):29-30, 33-38.

19. Core Team R. R: A language and environment for statistical computing Vienna, Austria: R Foundation for Statistical Computing; 2011.

20. Hartigan JA, Wong MA. Algorithm AS 136: A K-means clustering algorithm. J R Stat Soc Ser C Appl Stat. 1979;28(1):100-108.

21. US Census Bureau. Geographic Terms and Concepts - 2010 Census Divisions and Census Regions. 2017.

22. Prevalence of adult vision impairment and age-related eye diseases in America. National Eye Institute (NEI): Statistics and Data. 2016.

23. CDC Centers for Disease Control and Prevention. Vision Health Initiative (VHI). National Data. 2018.

24. Doran M. Saving ophthalmology's endangered subspecialties. Eye net. 2009;13(9):49-53.

25. Dhiman R, Singh D, Gantayala SP, et al. Neuro-ophthalmology at a tertiary eye care center in India. J Neuroophthalmol. 2018;38(3):308-311.

26. Acharya $\mathrm{N}$ et al. Incidence and prevalence of uveitis. Results from the pacific ocular inflammation study. JAMA Ophthalmol. 2013;131(11):1405-1412.

27. Lim SA, Wong WL, Fu E, et al. The incidence of neuro-ophthalmic diseases in Singapore. Ophthalmic Epidemiol. 2009;16(2):65-73.
28. Adelman RA, Nwanze, CC. The impact of the economy and recessions on the marketplace demand for ophthalmologists (an American Ophthalmological Society Thesis). Trans Am Ophthalmol Soc. 2011;109:49-65.

29. Lee PP, Hoskins HD, Parke DW. Access to care: eye care provider workforce 2007;125(3):406-410.

30. U.S. Census Bureau. Quick Facts. Population. Population, percent change. 2016.

31. Desal A, Stetzer J, Desai S, et al. Emerging Subspecialties in neurology: Neuro-ophthalmology. Neurology. 2016;86(21):e222-e224.

32. Parke DW. In the pursuit of fellowship. American Academy of Ophthalmology. 2015.

33. Bechtee B. Ocular Surgery News U.S. Edition. Growing demand for eye care services may highlight shortage of ophthalmologists. 2010 .

34. Gedde, SJ, Budenz DL, Pyaman H, et al. Factors influencing career choices among graduating ophthalmology residents. Ophalmol. 2005;112(7):1247-1254

35. Lee PP, Relles DA, Jackson CA. Subspecialty distributions of ophthalmologists in the workforce. Arch Ophthalmol. 1998;116(7):917-920. 